\title{
Gold Filled Products in the U.S.A.
}

\author{
FEDERAL REGULATIONS AND THEIR IMPACT
}

\section{S.L. Cantor}

Gold Filled Manufacturers Association, Attleboro, MA., U.S.A.

\begin{abstract}
Mechanically clad gold finishes are applied to a large variety of decorative products, affording them an attractive appearance, tarnish resistance and wearability. In the U.S.A. such finishes are subject to stringent Federal regulations, a fact which may well account for their major share of the U.S. decorative gold coatings market.
\end{abstract}

A very interesting article on the mechanically bonded and electroplated decorative gold industries in the U.K. and, to a lesser extent, Europe was recently published in this journal (1). It discussed the relative merits of electroplated and rolled gold products in those areas, and expressed the view that the application of compulsory standards for quality gold electroplate by a recognised authority would be to the benefit of the decorative and jewellery industries in many countries'. The present paper discusses aspects of the gold filled (mechanically bonded) decorative market in the U.S.A. with particular reference to the stringent quality specifications existing therein.

\section{Definition of Gold Filled}

The name originated from its earliest applications in which a sheet of carat gold was wrapped around a base metal rod, soldered and fused to it, and then the composite was drawn into a wire. Thus the wire was produced from 'gold filled with supporting metal', hence the term now in general use for the material. (2)

Use of the term gold filled in the U.S.A. is restricted by the Federal Trade Commission (FTC) to the description of products in which a layer of a gold alloy of a caratage of 10 or higher is mechanically bonded to all significant surfaces of a reinforcing substrate material. The carat gold must comprise at least $1 / 20$ th by weight of the total metal content of the product being described. Gold filled is generally produced in three categories of material, namely, sheet or flat stock, wire and tubing .

A product which contains mechanically bonded carat gold and conforms to all of the aforementioned prerequisites except that of the proportionate weight of its carat gold content (at least $1 / 20$ th) falls into the rolled gold plate category. As no minimum quantity of gold is required when identifying a product as rolled gold plate, the manufacturer is directed to precede disclosure of the gold fineness with a fraction which is accurately indicative of its carat gold content. Examples of this are 1 / 40th 12 K R.G.P. and 1/60th 14K R.G.P.

Other terminologies which may be used to describe rolled gold plate are gold overlay and gold plate. However, the gold fraction must always be disclosed and the term gold overlay may not be abbreviated.

It should be particularly noted that the term gold filled may be used only when the material conforms to the minimum quality prescribed. It may never be used to describe a product made to the rolled gold plate quality.

Thus Foster, (1), refers to flat stock in which the base metal is between two layers of bonded gold, for example, 70 per cent of the gold being on one side and 30 per cent on the other or, perhaps a given quantity of gold is divided equally on both sides of the material. In such situations in the U.S.A. many manufacturers insist upon $1 / 20$ th quality on each of the bonded surfaces which results in a product with $1 / 10$ th gold fraction overall, described as $1 / 10$ th $14 \mathrm{~K}$ gold filled when that particular caratage of gold is used. Many products of this type are made, particularly in the medallic art and coinage industries.

\section{Gold Filled Production}

The many gold filled decorative articles produced are made from three basic stock forms. Cigarette cases, lighters, watch cases, bracelets, lockets, cuff links, medals, charms and other bulk items are made from flat stock, whereas writing instruments, hollow bracelets, lipstick cartridges and similar shapes are produced from tubing. Gold filled wire is the basis for articles such as charms, optical frames, rings and collar pins. The production of the three basic shapes used in the manufacture of gold filled parts involves various techniques (2).

\section{Flat Stock}

In batch operations the gold alloy selected for carat value, strength, colour and other properties is cast into ingot form, rolled to a given thickness of sheet and placed on top of a bar of supporting base metal the mass of which is in a predetermined ratio to that of the gold alloy. Between these is a thin sheet of high temperature solder. The layers are then heated and pressed together so that the two alloys are fused into a single bar. After cleaning, the gold filled bar is fed through precision designed rolls under pressure and annealed to give flat stock with a consistent thickness of carat gold layer. In continuous operation, strip is also produced on a large scale by lamination of strips of base metals and of gold or gold alloy in a hot rolling mill.

\section{Tubing and Wire}

In one procedure, a disc of flat stock with gold on one side is placed gold side down on a steel die over a circular indentation. The stock is forced into the die by a plunger and formed into a wide shallow cup. After subsequent shaping and cupping in 


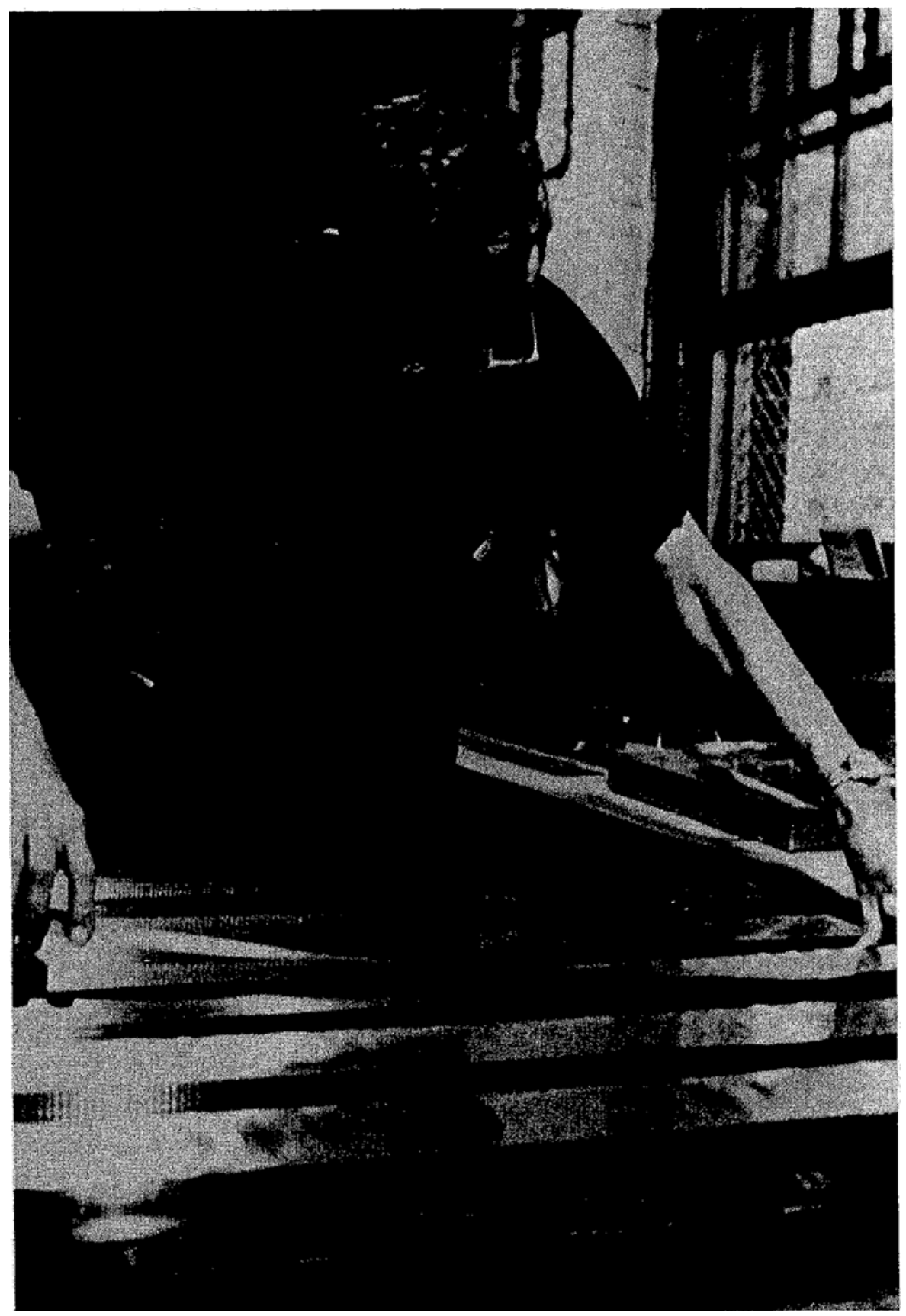

In the production of gold filled flat stock sheets of carat gold are carefully rolled to a given thickness and measured against bars of reinforcing base metal. A layer of solder is introduced between the two alloys which are fused into a composite bar under heat and pressure, with the gold layer on one or both sides of the base metal

required size. In an alternative procedure, suitable for larger scale production, gold alloy tube is sunk over tube or rod of base metal in relatively long lengths and the composition then processed to tube or wire by conventional means.

\section{Electroplated and Gold Filled Products}

Unlike specifications in many other countries for mechanically clad and plated gold, in addition to the requirements to which articles described respectively as gold filled, rolled gold and gold overlay must conform, the FTC also lays down certain legal minimum specifications which articles described as heavy gold electroplated, electroplated, gold wash or gold flash must meet. Thus a product upon which there has been applied by electrolysis a deposit of 10 carat gold or better, namely gold electroplate, cannot be so described unless there has been applied the equivalent of 7 millionths of an inch of fine gold (just less than $0.2 \mu \mathrm{m}$ ). If termed 'heavy gold electroplate' there shall be present the equivalent of 100 millionths of an inch $(2.5 \mu \mathrm{m})$ of fine gold. To emphasize the restrictiveness of this disclosure a product electrodeposited with an 18 carat gold alloy and marked 'heavy gold electroplate' would require a deposit of about 133 millionths of an inch in order to fulfil these

special draw presses and drawing over hardened steel arbors through a series of dies, a long seamless tube is produced which has a uniformly thick, hard, durable gold alloy surface. This may be used both as such and in the production of wire. For the latter purpose it is fitted with a central base metal core and fused to it to form a clad tod which is then reduced by either swaging or rolling in special wire reducing mills. Using multiple diamond die wire drawing machines the wire is then reduced to the requirements, that is a little less than $3.3 \mu \mathrm{m}$.

The U.S. FTC regulations state that an item which has less than 7 millionths of an inch (just less than $0.2 \mu \mathrm{m}$ ) of gold deposit may be described as gold wash or gold flash. There is no expressed obligation to disclose the carat fineness of its gold content. The belief expressed in (1) that these and similar deposits are unsatisfactory for consumer use is one with which the author is in full agreement. 
There are many items, particularly those which are fashioned by casting and then subsequently electroplated, which cannot be produced using gold filled or other mechanically bonded materials (1). However, it should be pointed out that even though there is significant limitation of design potential when using gold filled materials, the process of mechanical bonding is generally not performed by the manufacturer of the finished product but by a skilled specialist contractor. Therefore, the fidelity of a mechanical bond is guaranteed by expert workmanship and, in the U.S.A.; Federal requirements regarding quantity of gold used provide both retailers and distributors with an assurance of product quality.

As regards relative merits it is often inferred that the hardness of electrodeposits is greater than that of wrought alloys and gives them a correspondingly better resistance to wear. While it is true that gold electroplate is often harder than gold filled yielding higher abrasion resistance, the ductility of the former, a very important coating property, is invariably less than that of the latter. It should be pointed out that, in fact, there is no proven relationship between hardness and wear resistance of electroplated gold deposits. It has been shown empirically that specific items plated with gold of various hardnesses (and from different electrolytes) which showed up well in failure testing tended to show an optimum hardness range of 130 to 160 $\mathrm{kg} / \mathrm{mm}^{2}$ (Knoop).

A serious inherent problem suffered by gold electrodeposits is the presence of pores in thin layers, leading to corrosion or tarnishing of the base metal. The corrosion resistance of an electroplated article can consequently only be improved by increasing the thickness of the more noble component, the gold deposit. This solution is, of course, limited by the permissible cost range of the article, which might well ensure that the finished coating cannot be guaranteed to be pore-free. Over 20 years ago Schumpelt summed up the problem of porosity in noble metal coatings quite succinctly:

'No matter how few or how small, these pores are the breaches in the armor through which the enemy infiltrates. Once an attack has started, galvanic action will do the rest and undermine the whole surface.' (3)

Even today, porosity in gold electroplate and the necessity to guard against it require constant vigilance, particularly in view of the present trend towards reducing deposit thickness for economic reasons.

In any discussion of the relative merits of gold filled and gold electroplate the basic difference in the bonding of the interfacial region between gold and base metal must be emphasized. The superior adhesion exhibited by clad alloys, for example the 12 and $14 \mathrm{~K}$ gold filled commonly used in the U.S.A., stems from the nature of the manufacturing process steps used, that is, melting, fusion and rolling pressure which result in lack of porosity, high density and compactness of the gold layer and controlled diffusion of gold and base metal atoms. This results in a metallurgically bonded region in which no clear interface or potential plane of cleavage exists. In an as-electrodeposited gold coating a definite interface exists and can be seen readily in a microscope. This is a situation of potentially poor adhesion and if, for example, the base metal is passive, the gold electrolyte poorly maintained or the plating conditions are not correct, blistering or peeling of the gold layer can occur

Of further significance is the fact that many electrodeposited gold 'alloys' are not true equilibrium alloys and have compositions and properties which are very sensitive to changes in deposition conditions.

\section{Concluding Remarks}

It is clear that for both coating techniques strong quality specifications, preferably at Federal or Government level, are essential to ensure satisfactory meaningful standards of production and stimulation of markets. For gold filled articles in the U.S.A., FTC regulations and the Federal Stamping Law go a long way towards achieving these goals and are, in the author's opinion, largely responsible for the wide acceptance of these products in the U.S. market.

In conclusion it can be said that carat gold, gold filled and gold electroplate all have their place in the family of decorative and functional products. Each product type is unique and tends, by virtue of properties and cost considerations, to find its own market applications.

\section{References}

1 A. J. Foster, Gold Bull., 1982, 15, (2), 64-68

2 Anon., Am. Jew. Manuf, , 1980, Apr., 39-42

3 K. Schumpelt, Am.Jew: Manuf., 1960, Feb., 10-14 